\title{
Five Dimensional Spherical Symmetric Universe in Creation Field Cosmology
}

\author{
U.K. Panigrahi ${ }^{1}$, R.N. Patra ${ }^{2}$ and Mita Sharma ${ }^{3}$ \\ ${ }^{I}$ Ex. Principal, Academy Of Technocrats, Berhampur, Odisha, India. \\ ${ }^{2}$ Lecturer in mathematics, $P G$ Department of Mathematics, Berhampur University Berhampur, Odisha, India. \\ ${ }^{3}$ Department of Mathematics and Humanities, College of Engineering and Technology, Bhubaneswar, Odisha, \\ India.
}

\begin{abstract}
We have studied the Hoyle-Narlikar C-field cosmology with spherical symmetric five dimensional metric space time. Using methods of and Padmanabham [1], the solutions have been studied when the creation field $C$ is a function of time tonly. The geometrical and physical aspects for model are also examined.
\end{abstract}

\section{Introduction}

The phenomenon of expanding universe, primordial nucleon-synthesis and the observed isotropy of cosmic microwave background radiation (CMBR) are the three very important observations in astronomy. These were successfully explained by big-bang cosmology based on Einstein's field equations. Smoot et al. [2] revealed that the earlier prediction of the Friedman-Robertson-Walker type of models do not always exactly match with our expectations. Some puzzling results regarding the red shifts from the extra galactic objects continue to contradict the theoretical explanations given from the big-bang type of the model. Also, CMBR discovery did not prove it to be outcome of a big-bang theory. In fact, Narlikar et al. [3] have proved the possibility of non-relic interpretation of CMBR. To explain such phenomenon, many alternative theories have been proposed time to time. Hoyle [4], Bondi and Gold [5] proposed steady state theory in which the universe does not have singular beginning nor an end on the cosmic time scale. To overcome this difficulty Hoyle and Narlikar [6] adopted a field theoretic approach by introducing a mass less and charge less scalar field $\mathrm{C}$ in the Einstein-Hilbert action to account for the matter creation. In the C-field theory introduced by Hoyle and Narlikar there is no big-bang type of singularity as in the steady state theory of Bondi and Gold [5]. A solution of Einstein's field equations admitting radiation with negative energy mass less scalar creation field $\mathrm{C}$ was obtained by Narlikar and Padmanabhan [1]. The study of Hoyle and Narlikar theory [6-8] to the space times with higher dimensions was carried out by Chaterjee and Banarjee [9]. RajBali and Tikekar [10] studied C field Cosmology with variable $\mathrm{G}$ in the flat Friedman-Robertson-Walker model and with non flat FRW space time by Raj Bali and Kumawat [11]. The solutions of Einstien's field equations in the presence of creation field have been obtained for different Binachi type universes by Singh and Chaubey [12]. In the present work, we have studied the Hoyle and Narlikar C-field cosmology in spherical symmetric space time. We have assumed that $C(x, t)=C(t)$ that is the creation field $\mathrm{C}$ is a function of time' $\mathrm{t}$ ' only.

\section{Hoyle and Narlikar C-Field Cosmology}

Introducing a mass less scalar field called as creation field namely C-field, Einstein's field equations are modified. Hoyle and Narlikar field equations are

$R_{i j}-\frac{1}{2} g_{i j} R=-8 \pi\left({ }^{\mathrm{m}} T_{i j}+{ }^{\mathrm{c}} T_{i j}\right)$

Where ${ }^{\mathrm{m}} T_{i j}$ is matter tensor of Einstein theory and ${ }^{\mathrm{c}} T_{i j}$ is matter tensor due to C-field which is given by

${ }^{\mathrm{c}} T_{i j}=-f\left(C_{i} C_{j}-\frac{1}{2} g_{i j} C^{k} C_{k}\right)$

Where $f>0$ and $C_{i}=\frac{\partial C}{\partial x^{i}}$

Because of negative value of $T^{00}\left(T^{00}<0\right)$, the $\mathrm{C}$-field has negative energy density producing repulsive gravitational field which causes the expansion of the universe. Hence, the energy conservation equation reduces to

${ }^{\mathrm{m}} T_{; j}^{i j}=-{ }^{\mathrm{c}} T_{; j}^{i j}=f C^{i} C^{j}{ }_{; j}$

i.e. Matter creation through non zero left hand side is possible while conserving the overall energy and momentum.

Above equation is similar to

$m g_{i j} \frac{d x^{i}}{d s}-C_{j}=0$ 
Which implies that the 4-momentum of the created particle is compensated by the 4-momentum of the C-field. In order to maintain the balance, the $\mathrm{C}$-field must have negative energy. Further, the $\mathrm{C}$-field satisfy the source equation $f C^{i}{ }_{i j}=J^{i}{ }_{i j}$ and $J^{i}=\rho \frac{d x^{i}}{d s}=\rho v^{i}$, where $\rho$ is homogeneous mass density.

\section{Metric and Field Equations}

We consider the five dimensional spherical symmetric metric

$d s^{2}=d t^{2}-e^{\lambda}\left(d r^{2}+r^{2} d \theta^{2}+r^{2} \sin ^{2} \theta d \phi^{2}\right)+e^{\mu} d y^{2}$

Where $\lambda$ and $\mu$ are function of $\mathrm{t}$.

We assume $C(x, t)=C(t)$ and that

${ }^{\mathrm{m}} T_{j}^{i}=\operatorname{diag}(\rho,-p,-p,-p,-p)$

We have also assumed that the velocity of light and the gravitational constant are equal to one unit.

The field equations for metric (5) lead to

$\frac{3}{4} \dot{\lambda}^{2}+\frac{3}{4} \dot{\lambda} \dot{\mu}=8 \pi\left(\rho-\frac{1}{2} f \dot{C}^{2}\right)$

$\ddot{\lambda}+\frac{1}{2} \dot{\lambda} \dot{\mu}+\frac{3}{4} \dot{\lambda}^{2}+\frac{1}{2} \ddot{\mu}=8 \pi\left(-p+\frac{1}{2} f \dot{C}^{2}\right)$

$\frac{3}{2} \ddot{\lambda}+\frac{3}{2} \dot{\lambda}^{2}=8 \pi\left(-p+\frac{1}{2} f \dot{C}^{2}\right)$

$\dot{\rho}+\left(\frac{3 \dot{\lambda}+\dot{\mu}}{2}\right)(\rho+p)=f \dot{C}\left[\ddot{C}+\left(\frac{3 \dot{\lambda}+\dot{\mu}}{2}\right) C\right]$

Where (.) denotes the derivative with respect to 't' and $\dot{C}=\frac{d C}{d t}$ we can rewrite equation (10) as

$\frac{d}{d V}(V \rho)+p=f \dot{C}(V) \frac{d}{d V}[V C(V)]$

Where $V=e^{\left(\frac{3 \lambda+\mu}{2}\right)}$

In order to obtain a unique solution, one has to specify the rate of creation of matter energy (at the expense of the negative energy of the $\mathrm{C}$-field). Without loss of generality, we assume that the rate of creation of matter energy density is proportional to the strength of the existing C-field energy density. That is the rate of creation of matter energy density per unit proper volume is given by

$\frac{d}{d V}(V \rho)+p=\alpha^{2} \dot{C}^{2} \equiv \alpha^{2} g^{2}(V)$

Where, $\alpha$ is a constant and we have defined $\dot{C}(V) \equiv g(V)$.

From eqns.(11) and (13), we get

$g(V) \frac{d}{d V}(g V)=\frac{\alpha^{2}}{f} g^{2}(V)$

Which on integration gives $\quad g(V)=A_{1} V^{\frac{\alpha^{2}}{f}-1}$

Where, $A_{1}$ is an arbitrary constant of integration. We take the equation of state of matter as $p=\gamma \rho$.

Then from eqns. (13) and (15), we have

$\frac{d}{d V}(V \rho)+\gamma \rho=\frac{\alpha^{2}}{A_{1}{ }^{2}} V^{2\left(\frac{\alpha^{2}}{f}-1\right)}$

From eqn. (16)

$\rho=\frac{\alpha^{2} A_{1}{ }^{2}}{\left(2 \frac{\alpha^{2}}{f}-1+\gamma\right)} V^{2\left(\frac{\alpha^{2}}{f}-1\right)}$

$p=\gamma \frac{\alpha^{2} A_{1}^{2}}{\left(2 \frac{\alpha^{2}}{f}-1+\gamma\right)} V^{2\left(\frac{\alpha^{2}}{f}-1\right)}$

Now subtracting eqn. (8) from eqn. (9), we get

$\frac{d}{d t}\left[\frac{\dot{\lambda}-\dot{\mu}}{2}\right]+\left[\frac{\dot{\lambda}-\dot{\mu}}{2}\right]\left[\frac{3 \dot{\lambda}+\dot{\mu}}{2}\right]=0$

$\Rightarrow \frac{d}{d t}\left[\frac{\dot{\lambda}-\dot{\mu}}{2}\right]+\left[\frac{\dot{\lambda}-\dot{\mu}}{2}\right] \frac{\dot{V}}{V}=0$

Integrating the above equation, we get

$\frac{e^{\frac{\lambda}{2}}}{e^{\frac{\mu}{2}}}=k_{2} \exp \left(k_{1} \int \frac{1}{V} d t\right)$

Where $k_{1}$ and $k_{2}$ are constants of integrations.

In view of the fact that $V=e^{\frac{3 \lambda}{2}} e^{\frac{\mu}{2}}$ we write $\lambda(t)$ and $\mu(t)$ in the explicit form

$e^{\frac{\lambda}{2}}=D_{1} V^{\frac{1}{4}} \exp \left(X_{1} \int \frac{1}{V} d t\right)$

$\lambda(t)=2 \ln \left[D_{1} V^{\frac{1}{4}} \exp \left(X_{1} \int \frac{1}{V} d t\right)\right]$ 


$$
\begin{aligned}
& e^{\frac{\mu}{2}}=D_{2} V^{\frac{1}{4}} \exp \left(X_{2} \int \frac{1}{V} d t\right) \\
& \mu(t)=2 \ln \left[D_{2} V^{\frac{1}{4}} \exp \left(X_{2} \int \frac{1}{V} d t\right)\right]
\end{aligned}
$$

Where $D_{i}(i=1,2)$ and $X_{i}(i=1,2)$ satisfy the relation $D_{1}{ }^{3} D_{2}=1$ and $3 X_{1}+X_{2}=0$.

Using eqns. (7), (8) and (9) we get

$\frac{3}{2} \ddot{\lambda}+\frac{1}{2} \ddot{\mu}+\frac{9}{4} \dot{\lambda}^{2}+\frac{1}{4} \dot{\mu}^{2}+\frac{3}{2} \dot{\lambda} \dot{\mu}=\frac{16}{3} \pi(2-\gamma) \rho$

Now using equation (12) we get

$\frac{\ddot{V}}{V}=\frac{16}{3} \pi(2-\gamma) \rho$

From eqns. (17) and (27), we get

$\frac{\ddot{V}}{V}=\frac{16}{3} \pi(2-\gamma) \frac{\alpha^{2} A_{1}^{2}}{\left(2 \frac{\alpha^{2}}{f}-1+\gamma\right)} V^{2\left(\frac{\alpha^{2}}{f}-1\right)}$

This implies

$V=\left\{A_{1}\left(f-\alpha^{2}\right)\left[\frac{16 \pi(2-\gamma)}{3\left(2 \alpha^{2}-f-\gamma f\right)}\right]^{1 / 2}\right\}^{f} / f-\alpha^{2}{ }_{t}^{f} / f-\alpha^{2}$

From eqns. (15) and (29), we get

$g=\frac{1}{f-\alpha^{2}}\left[\frac{16 \pi(2-\gamma)}{3\left(2 \alpha^{2}-f-\gamma f\right)}\right]^{-1 / 2} \frac{1}{t}$

Also from equation, $\dot{C}(V)=g(V)$, we get

$C=\frac{1}{f-\alpha^{2}}\left[\frac{16 \pi(2-\gamma)}{3\left(2 \alpha^{2}-f-\gamma f\right)}\right]^{-1 / 2} \log t$

From eqns. (17) and (29), the homogeneous mass density becomes

$\rho=\frac{3 \alpha^{2} f}{16 \pi(2-\gamma)\left(f-\alpha^{2}\right)^{2}} \frac{1}{t^{2}}$

Hence from eqn. (18)

$p=\frac{3 \alpha^{2} \gamma f}{16 \pi(2-\gamma)\left(f-\alpha^{2}\right)^{2}} \frac{1}{t^{2}}$

From eqns. (32) and (33), it is observed that

1) When time, $t \rightarrow \infty$, we get, density and pressure tending to zero i.e., the model reduces to vacuum.

2) When, $f=\alpha^{2}$, there is singularity in density and pressure.

3) There is also singularity in density and pressure for $\gamma=2$.

Using eqns. (23), (25) and (29), we can obtain

$\lambda(t)=2 \ln \left[D_{1} k^{1 / 3} t^{\frac{f}{3\left(f-\alpha^{2}\right)}} \exp \left\{\frac{x_{1}}{k}\left(1-\frac{f}{\alpha^{2}}\right) t^{\frac{\alpha^{2}}{\alpha^{2}-f}}\right\}\right]$

$\mu(t)=2 \ln \left[D_{2} k^{1 / 3} t^{\frac{f}{3\left(f-\alpha^{2}\right)}} \exp \left\{\frac{x_{2}}{k}\left(1-\frac{f}{\alpha^{2}}\right) t^{\frac{\alpha^{2}}{\alpha^{2}-f}}\right\}\right]$

Now the scalar expansion $\theta=4 H$

$\theta=\frac{4}{3} \frac{f}{\left(f-\alpha^{2}\right)} t^{-1}$

The mean anisotropy parameter $A$ is given by

$A=\frac{1}{4} \sum_{i=1}^{4}\left(\frac{\Delta H_{i}}{H}\right)^{2}$

$A=27 \frac{X_{1}^{2}}{k^{2}} \frac{\left(f-\alpha^{2}\right)^{2}}{f^{2}} t^{2\left(\frac{\alpha^{2}}{\alpha^{2}-f}\right)}$

The shear scalar $\sigma^{2}$ is

$\sigma^{2}=\frac{1}{2}\left[\sum_{i=1}^{4} H_{i}^{2}-4 H^{2}\right]=\frac{4}{2} A H^{2}$

$\sigma^{2}=6 \frac{X_{1}^{2}}{k^{2}} t^{\frac{2 f}{\alpha^{2}-f}}$

The declaration parameter $q$ is given by

$q=\frac{d}{d t}\left(\frac{1}{H}\right)-1$

$q=2-\frac{\alpha^{2}}{f}$

Where $k=\left\{A_{1}\left(f-\alpha^{2}\right)\left[\frac{16 \pi(2-\gamma)}{3\left(2 \alpha^{2}-f-\gamma f\right)}\right]^{1 / 2}\right\}^{f / f-\alpha^{2}}$

If $f>\alpha^{2}$ then for large $t$, the model tends to isotropic case. 
Case I: $\boldsymbol{\gamma}=\mathbf{0}$ (Dust)

\section{Particular Cases}

In this case, we obtain the values of various parameters

$g=\frac{1}{f-\alpha^{2}}\left[\frac{32 \pi}{3\left(2 \alpha^{2}-f\right)}\right]^{-1 / 2} \frac{1}{t}$

$C=\frac{1}{f-\alpha^{2}}\left[\frac{32 \pi}{3\left(2 \alpha^{2}-f\right)}\right]^{-1 / 2} \log t$

$\rho=\frac{3 \alpha^{2} f}{32 \pi\left(f-\alpha^{2}\right)^{2}} \frac{1}{t^{2}}$

$p=0$

$\lambda(t)=2 \ln \left[D_{1} k_{1}{ }^{1 / 3} t^{\frac{f}{3\left(f-\alpha^{2}\right)}} \exp \left\{\frac{x_{1}}{k_{1}}\left(1-\frac{f}{\alpha^{2}}\right) t^{\frac{\alpha^{2}}{\alpha^{2}-f}}\right\}\right]$
$\mu(t)=2 \ln \left[D_{2} k_{1}{ }^{1 / 3} t^{\frac{f}{3\left(f-\alpha^{2}\right)}} \exp \left\{\frac{x_{2}}{k_{1}}\left(1-\frac{f}{\alpha^{2}}\right) t^{\frac{\alpha^{2}}{\alpha^{2}-f}}\right\}\right]$

Now the scalar expansion is

$\theta=\frac{4}{3} \frac{f}{\left(f-\alpha^{2}\right)} t^{-1}$

The mean anisotropy parameter $A$ is given by

$A=27 \frac{X_{1}{ }^{2}}{k_{1}{ }^{2}} \frac{\left(f-\alpha^{2}\right)^{2}}{f^{2}} t^{2\left(\frac{\alpha^{2}}{\alpha^{2}-f}\right)}$

The shear scalar $\sigma^{2}$ is given by

$\sigma^{2}=6 \frac{X_{1}{ }^{2}}{k_{1}{ }^{2}} t^{\frac{2 f}{\alpha^{2}-f}}$

The declaration parameter $q$ is given by

$q=2-\frac{\alpha^{2}}{f}$

Where $k_{1}=\left\{A_{1}\left(f-\alpha^{2}\right)\left[\frac{32 \pi}{3\left(2 \alpha^{2}-f\right)}\right]^{1 / 2}\right\}^{f / f-\alpha^{2}}$

If $f>\alpha^{2}$ then for large t, the model tends to isotropic case.

Case II: $\gamma=\frac{1}{3}$ (Disordered Radiation Universe)

In this case, we obtain the values of various parameters

$\rho=\frac{9 \alpha^{2} f}{80 \pi\left(f-\alpha^{2}\right)^{2}} \frac{1}{t^{2}}$

$p=\frac{3 \alpha^{2} f}{80 \pi\left(f-\alpha^{2}\right)^{2}} \frac{1}{t^{2}}$

$\lambda(t)=2 \ln \left[D_{1} k_{2}{ }^{1 / 3} t^{\frac{f}{3\left(f-\alpha^{2}\right)}} \exp \left\{\frac{x_{1}}{k_{2}}\left(1-\frac{f}{\alpha^{2}}\right) t^{\frac{\alpha^{2}}{\alpha^{2}-f}}\right\}\right]$

$\mu(t)=2 \ln \left[D_{2} k_{2}{ }^{1 / 3} t^{\frac{f}{3\left(f-\alpha^{2}\right)}} \exp \left\{\frac{x_{2}}{k_{2}}\left(1-\frac{f}{\alpha^{2}}\right) t^{\frac{\alpha^{2}}{\alpha^{2}-f}}\right\}\right]$

Now the scalar expansion

$\theta=\frac{4}{3} \frac{f}{\left(f-\alpha^{2}\right)} t^{-1}$

The mean anisotropy parameter $A$ is given by

$A=27 \frac{X_{1}^{2}}{k_{2}^{2}} \frac{\left(f-\alpha^{2}\right)^{2}}{f^{2}} t^{2\left(\frac{\alpha^{2}}{\alpha^{2}-f}\right)}$

The shear scalar $\sigma^{2}$ is

$\sigma^{2}=6 \frac{X_{1}^{2}}{k_{2}{ }^{2}} t^{\frac{2 f}{\alpha^{2}-f}}$

The declaration parameter $q$ is given by

$q=2-\frac{\alpha^{2}}{f}$

Where $k_{2}=\left\{A_{1}\left(f-\alpha^{2}\right)\left[\frac{80 \pi}{3\left(6 \alpha^{2}-4 f\right)}\right]^{1 / 2}\right\}^{f / f-\alpha^{2}}$

If $f>\alpha^{2}$ then for large t, the model tends to isotropic case.

Case III: $\gamma=1$ (Zeldovich fluid or stiff fluid)

In this case, we obtain the values of various parameters 
$\rho=\frac{3 \alpha^{2} f}{16 \pi\left(f-\alpha^{2}\right)^{2}} \frac{1}{t^{2}}$

$p=\frac{3 \alpha^{2} f}{16 \pi\left(f-\alpha^{2}\right)^{2}} \frac{1}{t^{2}}$

$\lambda(t)=2 \ln \left[D_{1} k_{3}{ }^{1 / 3} t^{\frac{f}{3\left(f-\alpha^{2}\right)}} \exp \left\{\frac{x_{1}}{k_{3}}\left(1-\frac{f}{\alpha^{2}}\right) t^{\frac{\alpha^{2}}{\alpha^{2}-f}}\right\}\right]$

$\mu(t)=2 \ln \left[D_{2} k_{3}{ }^{1 / 3} t^{\frac{f}{3\left(f-\alpha^{2}\right)}} \exp \left\{\frac{x_{2}}{k_{3}}\left(1-\frac{f}{\alpha^{2}}\right) t^{\frac{\alpha^{2}}{\alpha^{2}-f}}\right\}\right]$

Now the scalar expansion

$\theta=\frac{4}{3} \frac{f}{\left(f-\alpha^{2}\right)} t^{-1}$

The mean anisotropy parameter $A$ is given by

$A=27 \frac{X_{1}^{2}}{k_{3}{ }^{2}} \frac{\left(f-\alpha^{2}\right)^{2}}{f^{2}} t^{2\left(\frac{\alpha^{2}}{\alpha^{2}-f}\right)}$

The shear scalar $\sigma^{2}$ is

$\sigma^{2}=6 \frac{X_{1}^{2}}{k_{3}^{2}} t^{\frac{2 f}{\alpha^{2}-f}}$

The declaration parameter $q$ is given by

$q=2-\frac{\alpha^{2}}{f}$

Where $k_{3}=\left\{A_{1}\left(f-\alpha^{2}\right)\left[\frac{8 \pi}{3\left(\alpha^{2}-f\right)}\right]^{1 / 2}\right\}^{f / f-\alpha^{2}}$

If $f>\alpha^{2}$ then for large $\mathrm{t}$, the model tends to isotropic case.

\section{Discussion}

For each case of $2 f<\alpha^{2}$, we get negative deceleration parameter indicating that the universe is accelerating. This observation is consistent with the present day observation. The universe becomes isotropic for large value of $\mathrm{t}$. For a small of $\mathrm{t}$, the ratio $\frac{\sigma}{\theta}$ tends to zero. Therefore, the model approaches to isotropy at late times. Also, we have observed that the matter density is inversely proportional to square of time t. we can interpret our result as that the matter is supposed to move along the geodesic normal to the surface $\mathrm{t}=$ constant. As the matter moves the further apart, it is assumed that more mass is continuously created to maintain the matter density. However, the matter density tends to zero when time will be infinitely large.

\section{Conclusion}

In this work the Hoyle-Narlikar C-field Cosmology has been studied in the five dimensional spherical symmetric metric. The solutions have been studied in the case when the creation field $\mathrm{C}$ is a function of time only. The geometrical and physical aspects have been studied in some particular cases.

\section{References}

[1]. J.V. Narlikar, T. Padmanabhan (1985) Phys. Rev. D 32, 1982.

[2]. G.F. Smoot, et al. (1992) Astrophys. J. 396, 21

[3]. J.V. Narlikar, et al. (2003) Astrophys. J. 585, 1.

[4]. F.Hoyle (1948) Mon. Not. R. Astron. Soc. 108,372.

[5]. H. Bondi, T. Gold (1948) Mon. Not. R. Astron. Soc. 108,252.

[6]. F. Hoyle, J.V. Narlikar (1966) Proc. R. Soc. (London) A290, 162.

[7]. F. Hoyle, J.V. Narlikar (1963) Proc. R. Soc. (London) A273,1.

[8]. F. Hoyle, J.V. Narlikar (1964) Proc. R. Soc. (London) A282,178.

[9]. S. Chatterjee, A. Banerjee (2004) Gen. Relativ. Gravit. 36,303.

[10]. RajBali, R.S. Tikekar (2007) Chin. Phys. Lett. 24 No. 11.

[11]. RajBali, M. Kumawat (2009) Int. J. Theor. Phys. 48.

[12]. T. Singh, R. Chaubey (2009) Astrophys. Space Sci. 321, 5. 